\title{
Thrombotic thrombocytopenic purpura in the presence of connective tissue disease and HIV infection: A diagnostic and therapeutic challenge in a resource- constrained setting
}

\author{
R Perumal,,${ }^{1,2}$ MB ChB, MPH; J A Marais, ${ }^{1}$ FCP (SA); S Brown, ${ }^{1}$ FCP (SA) \\ ${ }^{1}$ Department of Internal Medicine, School of Clinical Medicine, Nelson R Mandela School of Medicine, College of Health Sciences, University of \\ KwaZulu-Natal, Durban, South Africa \\ ${ }^{2}$ Centre for the AIDS Programme of Research in South Africa, Nelson R Mandela School of Medicine, College of Health Sciences, University of \\ KwaZulu-Natal, Durban, South Africa
}

Corresponding author: R Perumal (rubeshanperumal@gmail.com)

Thrombotic thrombocytopenic purpura (TTP) may occur in the presence of other confounding diseases. We present a case of TTP in a patient with connective tissue disease and HIV infection, in whom the diagnosis and management of TTP was challenging. It is important to understand the various underlying mechanisms that drive TTP in the presence of these comorbid diseases, so that an appropriate treatment strategy can be initiated. Our patient failed an initial trial of plasma infusion alone, but responded well to plasma exchange.

S Afr Med J 2016;106(6):585-588. DOI:10.7196/SAMJ.2016.v106i6.10274

Thrombotic thrombocytopenic purpura (TTP) is a multisystem disorder characterised by microangiopathic haemolytic anaemia and resulting diffuse microcirculatory collapse. The classic clinical description is a pentad of thrombocytopenia, haemolytic anaemia, fever, renal dysfunction and neurological impairment. Acquired TTP accounts for the vast majority of clinical cases, and may be classic idiopathic TTP or secondary TTP. There has been a growing recognition of the secondary causes of TTP, which include, inter alia, collagen vascular disease, bone marrow transplantation, malignancy, pregnancy, infections and drugs. HIV-associated TTP has become a particularly important secondary cause of TTP in high-HIV-burdened settings. The association of TTP and connective tissue disease (CTD) was first described in 1961 with a case of systemic lupus erythematosus complicated by TTP. Mixed CTD (MCTD), on the contrary, is rarely complicated by TTP, with only 14 cases being described in the literature to date. We describe the first case of TTP occurring in a patient with undifferentiated CTD and HIV infection, and the complexity of diagnosis and management in a resource-constrained setting.

\section{Case presentation}

A 46-year-old black African woman was admitted with complaints of malaise, lethargy,

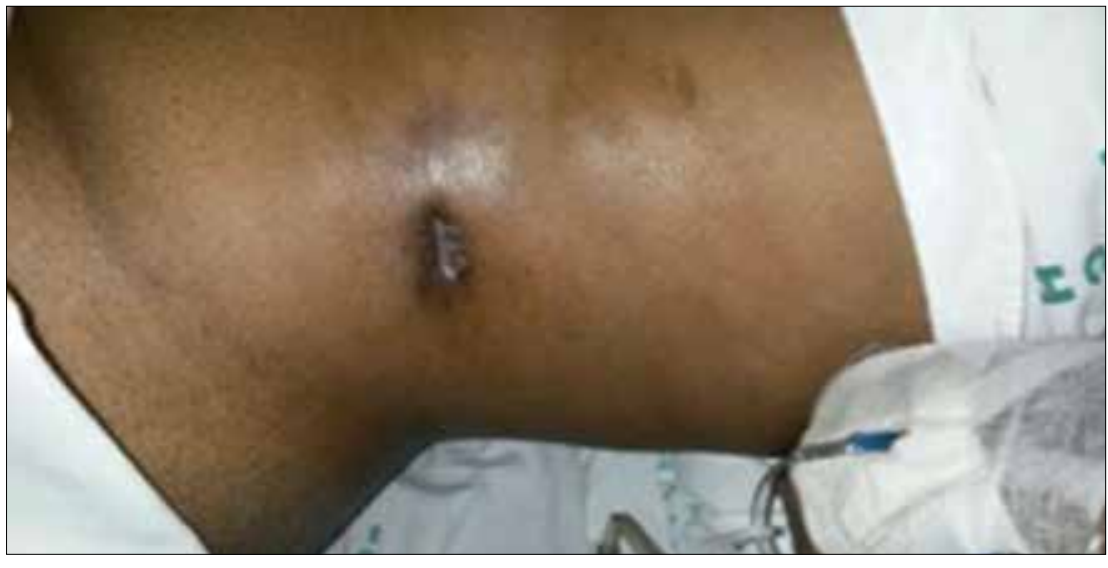

Fig. 1. Morphea or localised scleroderma.

fever, and headaches for a week. She was living with HIV, having been diagnosed in 2010, with a baseline CD4 count of 109 cells/ $\mu \mathrm{L}$. She was initiated on antiretroviral therapy (ART) within a month of her diagnosis and demonstrated a good response to therapy, with a suppressed viral load (VL) and a satisfactory rise in her CD4 count to 329 cells $/ \mu \mathrm{L}$ over 4 years. Two years prior to this admission, she had presented to the dermatology unit for investigation of a rash that she reported under both her breasts as well as over her abdomen. The presence of thickened plaque-like lesions was confirmed clinically. Histological examination revealed the lesions to be morphea, a form of localised scleroderma (Fig. 1).
On admission, she was ill-looking, pale, jaundiced and pyrexial (formal temperature of $38.3^{\circ} \mathrm{C}$ ). The patient's blood pressure was $126 / 82$, and her heart rate was 120 beats per minute. There was no Raynaud's phenomenon, sausage fingers, arthritis or myositis. There were no cutaneous manifestations of systemic lupus erythematosus (SLE) or features of systemic sclerosis. Examination of her respiratory system, cardiovascular system, nervous system and abdomen were normal. The full blood count revealed thrombocytopenia and normocytic normochromic anaemia (Table 1). A review of the peripheral blood film confirmed true thrombocytopenia, marked fragmentation of red blood cells, the extensive presence 


\section{Table 1. Initial laboratory findings}

\begin{tabular}{|c|c|}
\hline Test & Result \\
\hline \multicolumn{2}{|l|}{ Full blood count } \\
\hline White cell count (cells/L) & $13.98 \times 10^{9}$ \\
\hline Haemoglobin (g/dL) & 5.8 \\
\hline Cell volume (fl), mean & 93.1 \\
\hline $\begin{array}{l}\text { Cell haemoglobin }(\mathrm{pg}) \text {, } \\
\text { mean }\end{array}$ & 30.1 \\
\hline Platelet count (units/L) & $8 \times 10^{9}$ \\
\hline Peripheral smear & $\begin{array}{l}\text { Thrombocytopenia } \\
\text { Marked fragmentation of red cells } \\
\text { Extensive schistocytes } \\
\text { Polychromasia } \\
\text { Nucleated red cells }\end{array}$ \\
\hline LDH (U/L) & 3775 \\
\hline Total bilirubin $(\mu \mathrm{mol} / \mathrm{L})$ & 34 \\
\hline Coombs (direct) & Negative \\
\hline International normalised ratio & 1.0 \\
\hline PTT (s) & 30 \\
\hline Fibrinogen $(\mathrm{g} / \mathrm{L})$ & 3.7 \\
\hline Fibrin monomers & Negative \\
\hline Reticulocyte count, $n$ & 14.65 \\
\hline Corrected count, $n$ & 5.21 \\
\hline Reticulocyte production index & 1.7 \\
\hline Blood cultures & Negative \\
\hline Urine cultures & Negative \\
\hline $\begin{array}{l}\text { Sputum cultures (including } \\
\text { tuberculosis) }\end{array}$ & Negative \\
\hline $\operatorname{ESR}(\mathrm{mm} / \mathrm{hr})$ & $>140$ \\
\hline $\mathrm{CRP}(\mathrm{mg} / \mathrm{L})$ & 44 \\
\hline C3 (g/L) & 1.76 \\
\hline $\mathrm{C} 4(\mathrm{~g} / \mathrm{L})$ & 0.31 \\
\hline Anti-nuclear antibody & $\begin{array}{l}\text { Positive (titre 1:2 560, speckled } \\
\text { pattern) }\end{array}$ \\
\hline Anti-SSA(Ro) antibody & Positive \\
\hline Anti-URNP antibody & Positive \\
\hline Anti-DNA antibody & Negative \\
\hline Anti-Smith antibody & Negative \\
\hline Anti-SSB(La) antibody & Negative \\
\hline Anti-Jol antibody & Negative \\
\hline Anti-Scl70 antibody & Negative \\
\hline
\end{tabular}

of schistocytes, polychromasia and nucleated red blood cells. The anti-nuclear antibody test was positive at a titre of 1:2 560, as was the anti-U1RNP antibody, and anti-Ro antibody. Other specific antibodies including anti-DNA, anti-Smith, anti-CCP, anti-Jo1, antiLa, anti-Scl70, anti-centromere and antiphospholipid antibodies were negative. Raised lactate dehydrogenase and bilirubin levels, in the presence of a negative Coombs test, suggested the presence of non-autoimmune-mediated haemolytic anaemia. This was supported by a normal complement level. There was no evidence of sepsis or disseminated intravascular coagulopathy. Evaluation of iron studies, vitamin $B_{12}$ and folate were normal. There was mild renal impairment with an estimated glomerular filtration rate of $58 \mathrm{~mL} / \mathrm{min} / 1.73$ $\mathrm{m}^{3}$. Urinalysis was normal, and a pregnancy test was negative. The CD 4 count was 329 cells $/ \mu \mathrm{L}$, and the HIV VL was below detectable limits. The chest radiograph showed a normal cardiac shadow with normal lung fields. The clinical tapestry of thrombocytopenia, nonautoimmune haemolytic anaemia, fever and renal dysfunction led to the consideration of TTP as the leading differential diagnosis. While a thorough systematic attempt was made to investigate for potential secondary causes of TTP, two important secondary causes were clinically and biologically plausible - CTD-associated TTP and HIV-associated TTP. A flare of her CTD was an initial concerning differential diagnosis, but this became less likely as the results of normal complement, and evidence that the haemolytic process was non-autoimmune mediated, became available. Our initial treatment strategy for TTP included prednisone $1 \mathrm{mg} / \mathrm{kg} /$ day, fresh dried human plasma (FDP) $30 \mathrm{~mL} / \mathrm{kg} / \mathrm{day}$, red blood cell transfusion and gastric ulcer prophylaxis with omeprazole $20 \mathrm{mg}$ daily. She was monitored closely for signs of clinical or haematological failure, and was recognised to have deteriorated neurologically with seizure activity on day 3 - completing the classic clinical pentad of TTP. Failure of the treatment strategy was further confirmed by persistent thrombocytopenia and ongoing haemolysis manifest by declining haemoglobin and rising lactate dehydrogenase (Fig. 2). Plasmapheresis was promptly added to the treatment strategy (day 3), but required transfer to another facility with access to this resource. An improving clinical and laboratory trend was seen after 5 days of daily plasmapheresis therapy using fresh dried human plasma (FDP) as the exchange fluid. After a week of treatment, platelet count and haemoglobin increased, and there was no evidence of ongoing haemolysis.

\section{Discussion}

Thrombotic thrombocytopenic purpura is an important disease entity to consider in any patient presenting with thrombocytopenia and evidence of haemolytic anaemia. With improved access to healthcare and more timely diagnosis, it is now less common to see the classically described pentad of thrombocytopenia, haemolytic anaemia, fever, neurological manifestations and renal dysfunction. ${ }^{[1]}$ A more prudent diagnostic definition is that of microangiopathic haemolytic anaemia and thrombocytopenia, with or without renal failure or neurological abnormalities, and without another cause of thrombotic microangiopathy. The pathophysiology of TTP has become clearer with the discovery of ADAMTS13, a von Willebrand factor (VWF) cleavage enzyme. The central role of the protease, ADAMTS13, in the clinical expression of microcirculatory collapse has now been well established by laboratory studies that have demonstrated the propensity for microthrombosis in ADAMTS13 deficiency. ${ }^{[2]}$ VWF is a glycoprotein secreted by endothelial cells in response to endothelial injury, as large multimeric precursors referred to as unusually large VWF multimers (UL-VWFs). Without cleavage to smaller functional units, the UL-VWFs serve as potent surfaces for platelet aggregation and the bridging of platelets to the endothelium. It has therefore become apparent that the presence of an ADAMTS13 deficiency and the resulting excess of circulating UL-VWFs leads to disseminated platelet-rich thrombi, which block terminal arterioles. Microangiopathic haemolytic anaemia results from the mechanical destruction of red blood cells as they pass through these arterioles. The diffuse presence of thrombi in the arterioles results in microcirculatory collapse, which finds expression in the eventual ischaemic compromise of the organ systems in which it occurs. The predilection for collapse of the cerebral and renal microcirculations 


\begin{tabular}{|c|c|c|c|c|c|c|c|c|c|c|}
\hline & 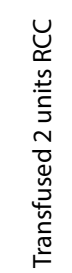 & & & 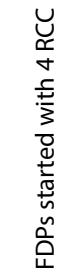 & & 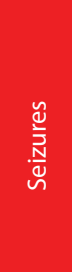 & 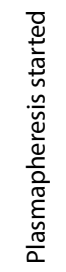 & & & \\
\hline & D0 & D1 & & D2 & & D3 & & D12 & D15 & D18 \\
\hline & $\mathrm{pm}$ & & $\mathrm{pm}$ & $\mathrm{am}$ & $\mathrm{pm}$ & $\mathrm{am}$ & $\mathrm{pm}$ & & & \\
\hline $\mathrm{Hb}$ & 5.8 & 9.1 & 5.2 & 3.8 & $t / f$ & $t / f$ & 7.9 & 9.6 & 12.3 & 11.9 \\
\hline LDH & 3775 & 3607 & & 3850 & 2926 & 3267 & 4717 & 348 & 234 & 220 \\
\hline Plts & 8 & 10 & 10 & 9 & & & 8 & 90 & 209 & 271 \\
\hline
\end{tabular}

Fig. 2. Disease evolution and treatment. ( $H b=$ haemoglobin; $L D H=$ lactate dehydrogenase; Plts = platelet count $; t / f=$ transfusion $F D P=$ fresh dried human plasma.$)$

is believed to be due to the high shear stress recorded in the vascular beds of these organs. However, the microcirculations of the heart, gastrointestinal tract and other organs have also been demonstrated to be potential sites of ischaemic compromise. ${ }^{[1]}$ Congenital TTP due to an inherited deficiency in ADAMTS 13 is exceedingly rare and seldom presents in adulthood. Idiopathic TTP is the most common form of TTP; it is an autoimmune disease characterised by the presence of auto-antibodies to ADAMTS13, which neutralise the cleavage enzyme and effectively result in reduced ADAMTS13 activity. However, it is important to investigate for secondary causes of TTP before the disease can be deemed to be idiopathic or classic TTP. A list of these secondary causes includes HIV, pregnancy-related disorders, drugs, malignancy and CTDs. While the mechanisms by which they result in TTP differ, they take common expression in a consumptive thrombocytopenia and a microangiopathic haemolytic anaemia. In addition, measurement on ADAMTS13 has not been shown to be useful for clinical decision-making in routine practice. Severe ADAMTS13 deficiency may not conclusively confirm the diagnosis of TTP, as more probable alternative diagnoses have been shown to exist in the presence of low ADAMTS13 levels.

There has been significant evolution in the treatments and outcomes for patients with TTP since the early 1900s. Corticosteroids, antiplatelet agents and splenectomy remained the primary methods of treatment until the mid-20th century, but resulted in the survival of only $10 \%$ of treated patients. ${ }^{[3]}$ The subsequent discoveries of plasma infusion, plasmapheresis (plasma exchange), and novel immune modulating therapies such as rituximab have resulted in a survival rate of nearly $90 \%$ in appropriately treated patients. ${ }^{[4]}$ Plasma infusion replenishes ADAMTS13 but does not remove ADAMTS13 antibodies from the patient's blood. Plasmapheresis, too, is able to replenish ADAMTS13, but is also able to replenish normal VWF multimers, remove ADAMTS13 antibodies, and remove unusually large VWF multimers from the patient's circulation. ${ }^{[5]}$ While adjuvant steroid therapy fails to confer additional survival benefit, it does reduce the requirements of both plasma infusion and plasma exchange. From the emerging novel therapeutic adjuvants, rituximab is a clear front-runner, with studies demonstrating the benefit of reducing the number and severity of relapses.

TTP associated with connective tissue was first documented in the scientific literature in 1961, in a case of SLE-associated TTP. Since then there have been just under 100 reported cases of SLE-associated TTP, which still represents the most common CTD association with TTP. TTP complicates SLE in only $0.7 \%$ of all SLE cases, and usually occurs in a patient with an established diagnosis of SLE. ${ }^{[6]}$ In contrast to the SLE, the association of MCTD and TTP has only been represented in the literature by 15 case reports. There have been no previous reports of TTP complicating undifferentiated CTD. Although an indistinct clinical entity, undifferentiated CTD has been recognised as an important diagnosis in a patient with symptoms and signs of a CTD that do not fulfil the criteria for one of the more established connective tissue diagnoses. ${ }^{[7]}$ In a prospective observational study, nearly $70 \%$ of patients with a baseline diagnosis of undifferentiated CTD maintained the same diagnosis after 10 years, making a clear case for the disease's recognition as an independent entity. ${ }^{[8]}$ The majority of the remaining patients went on to fulfil the criteria for SLE over the 10 years of follow-up, suggesting that for this group of patients the diagnosis of undifferentiated CTD 10 years prior represented the evolution of early SLE into a clinically recognisable form. The underlying pathophysiological basis for TTP occurring in these CTDs is believed to be the presence of antibodies against ADAMTS13, complement activity against ADAMTS13 and immune-mediated endothelial injury ${ }^{[9]}$ It is important to identify the features of TTP in the presence of CTD, as mortality approximates $100 \%$ if appropriate treatment is not initiated promptly. ${ }^{[10]}$ Haemolytic anaemia, thrombocytopenia, fever, neurological features and renal dysfunction may all be erroneously attributed to a CTD flare, especially in the case of SLE where a lupus flare may present in this exact way. Important distinguishing features that may assist in diagnosing TTP in this context include the presence of schistocytes on peripheral smear, a negative Coombs test, normal complement levels, and a low level of ADAMTS13 activity. ${ }^{[6]}$ In contrast, the absence of schistocytes, a positive Coombs test, low complement levels, and a normal level of ADAMTS13 activity suggest a CTDmediated haemolytic process rather than TTP. When the diagnosis of TTP is made in the presence of a CTD, treatment must include ongoing treatment of the CTD usually along with the use of steroids and cytotoxic agents. In addition, however, early plasmapheresis is essential to reduce mortality. ${ }^{[6,11,12]}$ Where plasmapheresis is not available, plasma infusion is a recommended albeit inferior alternative.

HIV-associated TTP represents a distinct clinical entity that is of great significance in regions of HIV hyperendemicity, where there are large numbers of untreated patients with severe immune deficiency. The aetiology of this entity is multifactorial, but led by the presence of a diffuse HIV vasculopathy. ${ }^{[13]}$ The resultant ongoing endothelial cell infection and injury stimulate the release of unusually large VWF from damaged endothelium. Importantly, absolute ADAMTS13 deficiency is not strongly implicated in the pathogenesis of HIV-associated TTP. ${ }^{[14,15]}$ However, the normal levels of ADAMTS13 in these patients is overwhelmed by the profuse secretion of UL-VWF. A relative 
deficiency of ADAMTS13 therefore occurs. This diffuse vasculopathy of HIV, and the resulting overwhelming of ADAMTS13 by UL-VWF secretion, characterises the occurrence of TTP in patients with advanced or untreated HIV. In these patients, there is emerging evidence that plasma infusion is non-inferior to plasma exchange, as the relative ADAMTS13 deficiency can be overcome by the infusion of plasma containing ADAMTS13. ${ }^{[16]}$ As there is limited, if any, role of antibodies to ADAMTS13, there is no need for the plasma exchange, which ordinarily has the benefit of being able to remove these antibodies. However, where plasma infusion is used alone, close monitoring for clinical or haematological failure is imperative. Evidence for microangiopathic haemolysis should be sought by monitoring platelet count, haemoglobin, lactate dehydrogenase and schistocyte measurements on the peripheral smear.

In patients with treated HIV, in whom the HIV VL is suppressed and cellular immunity has been restored, the occurrence of TTP is more likely to be due to the pathogenic mechanisms outlined for classic or idiopathic TTP, where the disease is led by antibodies to ADAMTS13 and therefore an absolute deficiency in ADAMTS13. ${ }^{[1,17-19]}$ In this group of patients with HIV, plasma exchange remains superior to plasma infusion. This is an important consideration, especially in settings where plasma exchange is a precious resource, and there is an inclination towards plasma infusion as the primary treatment modality for all HIV-positive patients, irrespective of their clinical, immunological or virological status. As greater numbers of patients with HIV become treated, the likelihood of the incident TTP being due to a diffuse HIV vasculopathy will be reduced. There must therefore be a similar paradigm shift in the determination of the primary treatment modality, with a lower threshold to offer plasma exchange.

In our case, the patient had a background history of HIV and CTD. It was crucial to distinguish between the possible causes of haemolytic anaemia in the presence thrombocytopenia, neurological impairment and renal impairment. The possibility of a CTD flare was considered alongside the possibilities of a CTD-associated TTP or HIV-associated TTP; a distinction being important as each possibility required a unique treatment strategy. The diagnosis of TTP was distinguished from haemolytic anaemia related to her CTD by means of the profuse presence of schistocytes on peripheral smear, normal complement levels and negative Coombs test. The HIV VL was suppressed and there was clinical and immunological improvement on ART. An initial trial of plasma infusion without plasma exchange was attempted as the literature supported this approach for HIVassociated TTP, and it has become common practice in our resourcelimited setting for HIV-associated TTP to be treated with a trial of plasma infusion alone before referral to central facilities with plasma exchange capabilities. Following the failure of this approach with our patient, plasma exchange was initiated and disease remission was achieved.
TTP in the presence of HIV and CTD presents challenging diagnostic and therapeutic dilemmas. A CTD flare can look similar to TTP, but even once a diagnosis of TTP is arrived at, the treatment strategy must be tailored according to the most likely underlying pathological mechanism of the TTP. In resource-limited settings, where HIVassociated TTP is more likely to be treated with a trial of plasma infusion alone, close monitoring for therapeutic failure must be performed so that appropriate escalation of therapy to plasma exchange can be initiated. A high index of clinical suspicion is necessary to make the diagnosis of idiopathic TTP in the presence of comorbidities, and early plasma exchange may be crucial to improve survival.

1. Scully M, Hunt BJ, Benjamin S, et al. Guidelines on the diagnosis and management of thrombotic thrombocytopenic purpura and other thrombotic microangiopathies. Br J Haematol 2012;158(3):323335. DOI:10.1111/j.1365-2141.2012.09167.x

2. Feys H, Roodt J, Vandeputte N, et al. Thrombotic thrombocytopenic purpura directly linked with ADAMTS13 inhibition in the baboon (Papio ursinus). Blood 2010;116(12):2005-2010. DOI:10.1182/ blood-2010-04-280479

3. George JN. How I treat patients with thrombotic thrombocytopenic purpura: 2010. Blood 2010;116(20):4060-4069. DOI:10.1182/blood-2010-07-271445

4. George J. Corticosteroids and rituximab as adjunctive treatments for thrombotic thrombocytopenic purpura. Am J Hematol 2012;87(Suppl 1):S88-S91. DOI:10.1002/ajh.23126

5. Rizzo C, Rizzo S, Scire E, et al. Thrombotic thrombocytopenic purpura: A review of the literature in the light of our experience with plasma exchange. Blood Transfus 2012;10(4):521-532. DOI:10.2450/2012.0122-11

6. Shah AA, Higgins IP, Chakravarty EF. Thrombotic microangiopathic hemolytic anemia in a patient with SLE: Diagnostic difficulties. Nat Clin Pract Rheumatol 2007;3(6):357-362. DOI:10.1038/ ncprheum0511

ncprheum 0511
7. Mosca M, Tani C, Bombardieri S. A case of undifferentiated connective tissue disease: Is it a distinct 7osca M, Tani C, Bombardieri S. A case of undifferentiated connective tissue disease: 1 s
clinical entity? Nat Clin Pract Rheumatol 2008;4(6):328-332. DOI:10.1038/ncprheum0799

8. Vaz CC, Couto M, Medeiros D, et al. Undifferentiated connective tissue disease: A seven-center crosssectional study of 184 patients. Clin Rheumatol 2009;28(8):915-921. DOI:10.1007/s10067-009-1175-2 9. Kuroda T, Matsuyama K, Nakatsue T, et al. A case of mixed connective tissue disease complicated with thrombotic thrombocytopenic purpura. Clin Rheumatol 2007;26(1):101-104. DOI:10.1007/s10067005-0105-1

10. Cheung WY. Thrombotic thrombocytopenic purpura and systemic lupus erythematosus - distinct entities or overlapping syndromes? Transfus Apher Sci 2006;34(3):263-266. DOI:10.1016/j. transci.2006.02.001

11. Souto Filho JT, de Barros PV, da Silva AM, Barbosa FA, Ribas GE. Thrombotic thrombocytopenic purpura associated with mixed connective tissue disease: A case report. Case Rep Med 2011:2011:953890. DOI:10.1155/2011/953890

12. Suzuki E, Kanno T, Asano T, et al. Two cases of mixed connective tissue disease complicated with thrombotic thrombocytopenic purpura. Fukushima J Med Sci 2013;59(1):49-55. DOI:10.5387/
thruki E, Kanno T, Asano T, et al. Two cases of mixed connective tissue disease complicated with thrombotic

13. Gunther K, Garizio D, Nesara P. ADAMTS13 activity and the presence of acquired inhibitors in human immunodeficiency virus-related thrombotic thrombocytopenic purpura. Transfusion 2007;47(9):1710-1716. DOI:10.1111/j.1537-2995.2007.01346.x

14. Malak S, Wolf M, Millot GA, et al. Human immunodeficiency virus-associated thrombotic microangiopathies: Clinical characteristics and outcome according to ADAMTS13 activity. Scand J Immunol 2008;68(3):337-344. DOI:10.1111/j.1365-3083.2008.02143.x

15. Blazes D, Decker C. Thrombotic thrombocytopenic purpura in HIV-infected patients. Infect Dis Clin Pract 2004;12(2):99-106. DOI:10.1097/01.idc.0000121025.62151.80

16. Novitzky N, Thomson J, Abrahams L, du Toit C, McDonald A. Thrombotic thrombocytopenic purpura in patients with retroviral infection is highly responsive to plasma infusion therapy. Br J Haematol 2005;128(3):373-379. DOI:10.1111/j.1365-2141.2004.05325.x

17. Becker S, Fusco G, Fusco J, et al. HIV-associated thrombotic microangiopathy in the era of highly active antiretroviral therapy: An observational study. Clin Infect Dis 2004;39 Suppl 5:S267-75.
. active antiretroviral

18. Coppo P, Bussel A, Charrier S, et al. High-dose plasma infusion versus plasma exchange as early treatment of thrombotic thrombocytopenic purpura/hemolytic-uremic syndrome. Medicine 2003;82(1):27-38. DOI:10.1097/00005792-200301000-00003

19. Hart D, Sayer R, Miller R, et al. Human immunodeficiency virus associated thrombotic thrombocytopenic purpura - favourable outcome with plasma exchange and prompt initiation of highly active antiretroviral therapy. Br J Haematol 2011;153(4):515-519. DOI:10.1111/j.13652141.2011.08636.x

Accepted 9 November 2015. 